\title{
Nasal Hygiene in the Treatment of Leprosy and the Use of Antimony.
}

F. Gordon Cawston.

7 HOUGH the relief of nasal catarrh is often associated with improvement of the health of otherwise normal patients, it would not appear that the importance of nasal hygiene has been sufficiently considered in the treatment of chronic diseases such as tuberculosis and leprosy, though the subject has repeatedly been emphasised by those in charge of consumptive homes and leprosy institutions.

Whether infection of the nasal passages should be regarded as primary focus of infection in leprosy or as secondary to a general infection, there can be no question that relief may be obtained by well regulated attempts to keep the nasal passages clear and relatively free from microbic infection, and that the secondary infection of nasal sinuses which are only too commonly associated with a simple nasal catarrh must lessen the chances of a patient overcoming a general infection.

Unfortunately, efforts to prevent infection during an epidemic of influenza have sometimes included the wholesale practice of washing the nasal cavity through with saline, thus tending to remove from normal persons the natural protection of the nasal mucus. Nasal lavage should be confined to persons suffering from infection of the nasal mucous membrane and, in these, a solution of sodium chloride or bicarbonate of soda is often of great service in removing infected matter and promoting the healing of ulcerated patches.

Nasal antiseptics are prepared as lotions, ointments and jellies. They should not be irritating to the mucous membrane and their use should not be unduly prolonged. It is conceivable that in using them one destroys microorganisms which are of service in the protection of the body, and that relative immunity to nasal infection may sometimes be more readily effected by anti-catarrhal vaccines employed either as nasal sprays or for repeated injection.

Antiseptic lotions which suggest themselves as useful for chronic nasal infection are hydrogen peroxide, glycothermaline, boracic and hexylresorcinol. The addition of glycerine is often of value, whilst rectified spirit may sometimes be added where there is a tendency to polypoid formation. 
As sprays, chloretone inhalant and hexylresorcinol are very useful indeed, whilst the occasional application of an antiseptic nasal ointment or jelly, such as ephregel or vix, renders nasal lavage less necessary and effects improvement by causing collapse of the turbinated bodies whose congestion prevents the ready escape of infected mucus or the normal ventilation of all parts of the nasal cavity.

Constant swelling of the turbinates may be produced by irritation during dust storms or in heated atmospheres with much humidity. Overclothing and over-eating aggravate the tendency, as does the presence of a deep-seated nasal infection. Where the nasal passages are kept clean and clear, many deep-seated nasal sinuses improve or may be cured without other measures being taken.

It is reasonable to suppose that careful attention to nasal infection in persons suffering from respiratory diseases and in cases of leprosy would enable the system more readily to deal with a chronic general infection. A leprologist tells me that he has obtained results which are definitely encouraging in a series of cases of leprosy treated with alkaline nasal douching and in the use of chloretone nasal sprays. Another informs me that he favours electrargol by spray in leprosy because of the definite improvement he has observed in ulceration of the nasal passages. I have myself observed improvement in the nasal condition of such cases whilst they were receiving a series of intramuscular injections of colloidal antimony. In the Leprosy Review for April, 1930, N. Pavloff mentions a $\mathbf{5}$ per cent. solution of chromic acid as particularly serviceable in the treatment of leprosy processes of the nose and throat.

Whilst a thorough investigation into the claims that are being made for nasal antiseptics in the control of respiratory disease is needed, there can be no doubt that'conservative measures for the relief of nasal catarrh might be more widely used among chronic sufferers and incurables. It would be of great service if those who are in charge of leprosy institutions could undertake an investigation into the relative value of those remedies which are found to be of service in the treatment of chronic infection of the nasal passages, and report how far the improvement of the nose and throat may be associated with an arrest of the progress of the disease or with general improvement of the patient's power of resistance.

It is to be regretted that many officials are not encouraged to publish in the medical press the result of their own careful researches, and that so much of the work that 
is being carried on is lost to science through being incorporated in inaccessible official returns.

I cannot find that antimony was used seriously with the object of overcoming the effects of leprosy previous to 1919, when the Secretary of the Indian Immigration Trust Board in Natal, with the concurrence of the two medical officers, granted me permission to treat a number of patients who were suffering from leprosy. The improvement shown in the general and local condition of these patients appealed to the authorities as well as to three medical men who had an opportunity of examining them, and it was on the recommendation of the Department of the Interior that I forwarded to Robbin Island a supply of the preparation of antimony which I had used in the treatment.

Other investigations which I have made on behalf of the British Medical Association convinced me that the most effective and least toxic preparation of antimony with which we are acquainted at the present time, is antimonium potassium tartrate given skilfully in carefully regulated doses in fresh solution intravenously, and that the injections should be given in the morning an hour or two after a light breakfast. I tested several others preparations of antimony including various colloidal preparations, but regarded all which might be administered orally or intramuscularly as somewhat uncertain in their action. About the same time, U. N. Brahmachari, in India, and R. G. Archibald, in the Sudan, recorded success from other preparations of antimony in the treatment of leprosy.

Unfortunately intravenous injections of antimonium potassium tartrate seem to have been responsible for undesirable consequences in the treatment of cases of leprosy in some countries, and this would seem to have been responsible for the discontinuance of antimony injections in the Amatikulu leprosy institution, where evident success was being obtained and where over 200 cases had received intravenous injections of an antimony preparation.

It is possible that local conditions have not been sufficiently considered in the reaction of leprous patients to treatment with antimony. When the beneficial effects of antimony injections in the treatment of leprosy in Natal had been published, the question was raised whether sufferers in Natal were affected with some parasitic infections which would reasonably respond to antimony therapy, irrespective of its influence on the leprosy bacillus. The late Sir Andrew Balfour, who was specially impressed with the return of sweating in leprotic areas among these Zululand 
patients whilst under treatment with antimony, drew my attention to the extraordinarily contradictory reports of its value which were being received from various parts of the world. There would seem to be no doubt that intravenous injections of tartar emetic given at so great an altitude as that of Maseru in Basutoland, had a detrimental effect on the cases, though I have used it with success on other patients who were not cases of leprosy, in Southern Basutoland, in a dry mountainous climate of over 5,000 feet above sea level.

Antimony is closely allied to arsenic and, in therapeutic doses, it should prove to be a good hepatic stimulant besides being a useful remedy for schistosomiasis and for many skin diseases. Vin. antimonialis is a very useful expectorant which largely has fallen out of use. Personally, I much prefer it to tinc. camph. co., as its action over a few days is usually far superior. I was much impressed with the confidence patients at Westfort, Pretoria, had in this expectorant, and I have sometimes felt that where intravenous injections of antimonium potassium tartrate cannot readily be given, it might be better to rely on the use of vin. antimonialis than on other antimony injections.

Several of the newer antimony preparations, though less to be desired than tartar emetic for the treatment of schistosomiasis in spite of a good antimony content, would appear to be specially indicated in chronic skin conditions, and, if it becomes accessible, a new preparation of fouadin, which is now being tested, in view of its calcium content, would seem to be indicated in the treatment of leprosy where there is skin ulceration or other indication for the use of antimony. I am interested to learn that this drug is being tested in the Dutch Indies for the relief of leprosy.

I have not been afforded the opportunity of officially treating leprous patients in the Union for the last 15 years or since I obtained the success I reported to the British Medical Association in 1919; but where antimony has been used in South African institutions on a large scale, it has sometimes proved more beneficial than chaulmoogra oil, and this result has been recorded officially.

The improvement which was noted at the Amanzimtoti institution whilst the cases were under treatment with antimony injections, was continued for several years and until this method of treatment was no longer sanctioned.

Racial predisposition to disease has received careful attention, but the fact that the therapeutic doses of any drug may vary at different altitudes and among different 
races seems to have been overlooked. When acting as medical officer under the Basutoland Government in 1931, during a prolonged winter drought, it seemed that almost any food or medicine had a beneficial effect in cases of deficiency disease. I found special use for injections of phosphorus, glucose, saline, and boiled sea-water and for tinc. rhei co., by the mouth. Intravenous injections of antimony, when called for, were difficult to administer because of the smallness of the superficial veins and the darkness of the skin, but I experienced no toxic effects. In Natal I have found Zulus able to tolerate large and repeated intravenous injections of antimonium potassium tartrate in fresh solution; the sodium salt is too frequently associated with shoulder pains and gastric disturbances for me to use it in private practice. Indians also tolerate larger doses than Europeans, but this may be due to the fact that they are small eaters. The toxic effects of antimony therapy which have been experienced in various parts of the world may possibly be due to unskilful regulation of the dose and to unreliable and stale solutions, but the idiosyncrasy of patients must be considered and, where a large number of patients have been found to respond to antimony therapy, it is unreasonable to condemn the use of the drug in that area because of unsatisfactory reports from its use in other countries.

Since antimony in the treatment of leprosy did not receive official approval in Zululand and was discontinued, official opinion has been guided by reports of its use in other countries and in other provinces of the Union and this may account for the recent official attitude towards its use.

In reply to a communication I am informed :-

"I am to state that antimony in its various forms has been given a thorough trial in our institutions, and that after such trial the Board was of opinion that the drug had no beneficial action whatever upon leprotic lesions. Its chief use was found to be in helping to clear up oldstanding trophic ulcers, but no effect upon either nodular or anæsthetic conditions was ever noticed."

When it is impossible to find a suitable vein for administering tartar emetic to a patient suffering from creeping eruption or other condition for which antimony is indicated, I usually employ one or two intramuscular injections of antimosan, fouadin, Crookes' colloidal antimony or Comar's combined antimony and sulphur in colloidal condition.

Unfortunately, the term " tartar emetic " has sometimes been applied to the sodium salt which, in my experience, 
is more commonly associated with toxic effects than tartar emetic. Where there is evident improvement in the general condition of a leprous patient, and where one observes, not only relief of the paralysis but healing of ulcerated patches and a return of sweating in anæsthetic areas, one is justified in continuing the use of the drug, but it must be administered with caution, and it is doubtful whether it should be given for more than a week or fortnight at a time, the general constitution being encouraged to exert itself in overcoming the infection.

\section{REFERENCES.}

(1) Cawston, F. G.- "Antimony in Leprosy," British Medical Journal 1920. December 4th, 1920.

1921. March 19th, 1921.

(2) Wildish, G. H._-“ Antimony in Leprosy," British Medical Journal. 1922. January 14th, 1922. 